\title{
Marc Debono, Langue et droit. Approche sociolinguistique, historique et épistémologique.
}

Préface d'Anne-Marie Chabrolle-Cerretini. Bruxelles :E.M.E. \& InterCommunications, 2013, 388 p. ISBN 978-2-8066-0770-6 (collection : «Proximités - Sciences du langage », dirigée par Philippe Blanchet).

\section{Henri Besse}

\section{(2) OpenEdition \\ Journals}

Édition électronique

URL : https://journals.openedition.org/dhfles/3645

DOI : $10.4000 /$ dhfles.3645

ISSN : 2221-4038

\section{Éditeur}

Société Internationale pour l'Histoire du Français Langue Étrangère ou Seconde

Édition imprimée

Date de publication : 1 juin 2014

Pagination : 200-204

ISSN : 0992-7654

Référence électronique

Henri Besse, " Marc Debono, Langue et droit. Approche sociolinguistique, historique et épistémologique. », Documents pour l'histoire du français langue étrangère ou seconde [En ligne], 52 | 2014, mis en ligne le 02 août 2016, consulté le 28 mai 2021. URL : http://journals.openedition.org/ dhfles/3645; DOI : https://doi.org/10.4000/dhfles.3645

Ce document a été généré automatiquement le 28 mai 2021.

(c) SIHFLES 


\section{Marc Debono, Langue et droit. Approche sociolinguistique, historique et épistémologique.}

Préface d'Anne-Marie Chabrolle-Cerretini. Bruxelles :E.M.E. \& InterCommunications, 2013, 388 p. ISBN 978-2-8066-0770-6 (collection : «Proximités - Sciences du langage », dirigée par Philippe Blanchet).

\section{Henri Besse}

1 L'ouvrage est la refonte, clarifiée et amendée, des deux premières parties d'une thèse soutenue en 2010. Sa singularité tient à ce que son auteur y a élaboré une réponse, à la fois personnelle et scientifiquement étayée, à une question qu'il s'était posée lors de ses études supérieures de droit (un DEA sur la censure cinématographique et un Diplôme universitaire de droit comparé), ce qu'il appelle « la question de la langue en droit ». Il s'y était étonné, les jugeant quelque peu "conservatrices", des "positions très affirmées et régulièrement réitérées de [s]es enseignants de droit" quant à «l'importance de la correction linguistique pour le juriste »(p. 10). Question qui a joué un rôle dans sa réorientation vers les sciences du langage et la didactique des langues, quand il fut chargé d'enseigner le «français juridique» à des étudiants non francophones. D'où une thèse qui confronte son passé de juriste à son présent d'enseignant-chercheur en français langue étrangère, et d'où l'acuité - quasi militante parfois, mais toujours formulée avec mesure - du présent ouvrage enté sur un vécu personnel et professionnel. N'en parle-t-il pas d'ailleurs comme d'une "recherche 'expérientielle'» (p. 9) ? Pourquoi publier un compte rendu de Langue et droit dans la revue de la SIHFLES ? Parce qu'il y est beaucoup question de l'histoire concomitante de la normalisation-unification, en quelque dix siècles, et de la langue et du droit français, et parce qu'une certaine historicité est au cœur de la sociolinguistique et de l'épistémologie qui le sous-tendent. Les trois adjectifs de son sous-titre ne se situent donc pas tout à fait sur le même plan: "l'historique " y étant partie prenante du « sociolinguistique » et de l'«épistémologique ». 
2 Langue et droit s'ouvre par une brève préface de A.-M. Chabrolle-Cerretini, auteur en 2007 d'une étude sur « la vision du monde de Wilhem von Humboldt », notion dont elle fait - bien qu'elle soit antérieure à l'émergence de la linguistique en tant que telle "un concept linguistique». Préface suivie d'un tout aussi bref «avant-propos» de l'auteur, qui y retrace son "parcours universitaire 'migratoire' et interdisciplinaire " (p. 9). Le corps de l'ouvrage proprement dit est divisé en deux grandes parties, encadrées par une courte introduction (p. 13-18) et par un encore plus court «élargissement conclusif» (p. 333-336). L'introduction souligne que, même si les affinités entre sciences du droit et sciences du langage ont été souvent évoquées, les recherches en «jurilinguistique» ou en "linguistique juridique» restent peu développées, en France particulièrement, l'auteur ayant donc dû œuvrer «dans un champ transfrontalier» encore "largement à défricher" (p. 15). Quant à "l'élargissement conclusif ", il se borne à orienter le lecteur vers les articles les plus récemment parus de l'auteur, articles où est repris le contenu, proprement didactique, de la troisième et dernière partie de sa thèse absente de Langue et droit.

En cent trente pages et cinq chapitres, la première partie traite des conceptions que l'on s'est faites en Europe, de la fin du XVIII ${ }^{\mathrm{e}}$ siècle à nos jours, des inévitables interrelations qu'entretiennent le domaine du droit et le domaine de la langue, le juridique. Debono les fait remonter à ce qu'il appelle - à l'instar de P. Sériot qui a dirigé, en 2008, un ouvrage intitulé: La question du déterminisme en Russie actuelle «l'humboldtianisme» (p. 23), ou bien - plus justement à notre avis - «l'idée herdérohumboldtienne» (p.125). À savoir, la ré-interprétation, par des jurisconsultes allemands, de certaines propositions "philosophiques" avancées par deux nonjuristes, J. G. von Herder, qui fut poète et théologien autant que philosophe, et surtout W. von Humboldt, qui consacra la fin de sa vie à une «linguistique comparée » alors émergente. Ré-interprétation juridique de propositions qui ne l'étaient donc pas originellement, et qui tend à négliger d'autres propositions de ces auteurs, en particulier chez Humboldt, à même de la nuancer ou de la contester. Elle revient à poser l'existence « d'un rapport strict de détermination de la pensée par la langue » (p. 31 ), thèse qualifiée de "déterminisme linguistique " quand elle est radicalement affirmée et de « relativisme linguistique » quand elle l'est plus prudemment.

Son chap. 1 rappelle que ce fut la thèse, à partir de 1814-1815, de jurisconsultes allemands tels F. C. von Savigny ou J. Grimm, dits de « l'École historique de droit ». Pour eux, le droit est d'abord une émanation populaire et spontanée du Volksgeist (« l'esprit du peuple»), conception qu'ils opposent à la rationalité universalisante du Code Napoléon dont les Allemagnes d'alors venaient juste de se libérer. Thèse qui sera radicalisée et racialisée, un siècle plus tard, par l'école Recht und Sprache (« droit et langue »), dont E. Forsthoff fut le plus illustre représentant.

5 Cet «humboldtianisme jurilinguistique » allemand n'est pas dissociable (chap. 2) de l'émergence de l'État-nation allemand tout au long du XIXe siècle et, durant la première moitié du XXe siècle. Et il en va de même de sa variante post-soviétique actuelle, évidemment liée au nationalisme d'une Russie désireuse de recouvrer sa puissance d'antan. Pour Debono - proche sur ce point de l'auteur de sa préface - ces "humboldtianismes" résultent de la confusion de deux notions que distinguait pourtant Humboldt: celle de Weltanschauung ("conception du monde», cadre de pensée qui vise à transcender par une réflexion universalisante la diversité des perceptions du réel selon les langues) et celle de Weltansicht ("vision du monde », telle 
qu'une langue l'impose spontanément à ceux qui la parlent), deux notions, dont l'une tempère l'autre, que ces "humboldtianismes" nationalistes réduisent à la seule Weltansicht, s'opposant ainsi tant à «l'universalisme » des Lumières qu'à tout droit « naturel » et partant universalisable.

Dans son chap. 3, Debono montre la persistance de ces "humboldtianismes » dans nombre de projets contemporains, entre autres certains de ceux qui visent à résoudre les difficultés que la diversité de ses vingt-trois langues "officielles» de l'Union européenne (UE) pose à l'élaboration d'un droit commun à ses vingt-huit membres. On se rappelle peut-être que l'académicien français Maurice Druon a publié, il y a dix ans, un manifeste "en faveur de la langue française comme langue juridique de l'Europe " qui, selon Debono, s'appuie contradictoirement sur "l'impérialisme universaliste rivarolien » et sur « l'exaltation herdéro-humboldtienne » (p. 56). «Tradition française de l'humboldtianisme» (p. 63) qu'il retrouve chez des grammairiens français tels J. Damourette et É. Pichon qui, partant de la langue (en l'occurrence, le français) pour montrer comme se forme la pensée, dériveraient «vers un racisme linguistique affirmé » (p. 64); ou tel G. Guillaume notant, à propos de l'article en français, que "l'état du nombre dans les langues est corrélatif à l'état de civilisation », ce qui serait tout aussi «clairement raciste» (p. 65). Pour lutter contre ces « humboldtianismes", l'auteur invite à " redécouvrir Humboldt pour penser le multilinguisme européen au $\mathrm{XXI}^{\mathrm{e}}$ siècle » (p. 81), tout en restant conscient que ce « réinvestissement de la pensée humboldtienne » n'est pas sans risque « pour penser la construction européenne » (p. 102).

7 Dans son chap. 4, sont analysées les résistances auxquelles on se heurte quand on cherche à s'opposer aux "politiques linguistiques à tendance déterministe» (p. 105), l'idée "déterministe » tirant sa force d'être "à la fois intuitive et indémontrable » (p. 118). Le regain des nationalismes dans l'UE atteste de sa force présente, tout comme les gender studies qui dénoncent le «machisme» des langues telles qu'ordinairement pratiquées. Debono en donne un bon exemple à propos de Mademoiselle dans les circulaires françaises de ces dernières années (p. 112-118).

8 Enfin le chap. 5 de cette première partie s'interroge sur les défis que pose le «plurilinguisme juridique européen » (p. 121) aux divers projets visant à l'uniformiser. Debono y opte pour une "uniformisation-processus ", qui serait le «fruit du contact permanent des langues et traditions au sein des institutions juridiques européennes ", contre une "uniformatisation-réalisation " imposée d'en haut et nécessairement «contrainte et artificielle»(p. 124). La «jurilinguistique canadienne», née de la cohabitation de deux langues et deux traditions juridiques, a développé une « hybridité jurilinguistique » qui lui paraît aller dans ce sens, tout comme l'expérience suisse de la "rédaction conjointe de textes législatifs» en français ou en allemand, ou l'introduction de traducteurs dans la rédaction même des lois, le «traducteur législatif » étant alors promu "rédacteur législatif». Contrairement à ce qu'avancent les tenants de l'hypothèse déterministe, ces pratiques plurilingues et plurilinguistiques sont, pour Debono, "source de sécurité juridique " parce que "plusieurs versions d'un même texte sont autant de gages d'une interprétation pertinente » (p. 143). Bref, à ses yeux, «la pratique du juriste européen est une pratique [qui devrait être] fondamentalement herméneutique et comparative» (ital. originales, p. 132). 

qu'il a menés, en 2007-2008, auprès d'enseignants-chercheurs de droit d'une université française, «analyse discursive herméneutisée » (p. 163) qui tranche avec la plupart des analyses de discours actuelles, en ce qu'elle n'écarte ni le "déjà là » ni le « en vue de " de celui qui la mène. Ce qui frappe Debono dans ces entretiens, c'est que n'y est évoqué « qu'à la marge, uniquement sous forme d'exception à un fonctionnement jugé normal et souhaitable", tout ce qui peut relever de «la dimension herméneutique du fonctionnement du langage et du droit » (p. 207). La sécurité juridique est, pour ses sept "témoins juristes", à rechercher d'abord dans «la stabilité du rapport signe/sens " (p. 211) tel que posé en français, ce qu'ils vérifient en consultant plus volontiers «le Littré » ou « le Robert », voire l'Histoire de la langue française de F. Brunot, que des études ou des dictionnaires plus récents. Quant à leur plurilinguisme et plurijuridisme, il est loin d'être évident, même si l'un d'entre eux affectionne les citations latines et s'il y est parfois question du Common law anglo-saxon.

Pour Debono (chap. 2 de cette seconde partie), cette détermination du droit français par la langue française est difficilement dissociable du «projet politique, relationnel, d'édification d'un État national » français (p. 247). Il en rappelle brièvement, de ce point de vue, les principales étapes, des Serments de Strasbourg (842) au Code Napoléon, " considéré comme l'aboutissement de l'entreprise d'unification du droit commencée au XIII siècle » et comme " une œuvre présentant une langue française 'parfaite' » (p. 241). «L'École de l'Exégèse », qui a dominé la conception et l'enseignement du droit en France au moins jusqu'au milieu du XXe siècle, s'inscrit dans ce même projet, dans cette « intrication de deux processus, historiquement concomitants» (p. 229) qui ont fini par faire « système », au point de rejeter dans le «non-droit » ou le «non-langue » tout ce qui n'y entre pas (p. 242).

"Système" dont traite, en plus de quatre-vingts pages, le dernier chapitre de l'ouvrage. Y est posée l'existence «d'un clivage épistémologique» qui divise tant les sciences du droit que les sciences du langage entre deux paradigmes, l'un dit sémiotique l'autre herméneutique, Debono optant pour une variante «modérée » (p. 296) du second afin de «dépasser l'interprétation déterministe » de la pensée par la langue (p. 249).

13 Si l'on s'est astreint à évoquer le contenu des huit chapitres de Langue et droit, c'est que l'on n'a pas voulu trop mutiler l'empan, diachronique et synchronique, des interrogations et des recherches que l'auteur y a mobilisées. Les quarante-trois pages de sa bibliographie - et pourtant deux ou trois des auteurs donnant lieu à citation ne s'y retrouvent pas - en attestent clairement. Les historiens « de métier » jugeront que les « sources primaires " y sont beaucoup moins présentes que les « secondaires », mais il ne fait pas de doute que Debono dispose des connaissances lui permettant de puiser aux premières pour conforter ou infirmer les secondes. Un regret toutefois, l'ouvrage ne comporte ni index des noms mentionnés ni index des notions traitées, ce qui n'en facilite pas la (re)consultation.

Reste une œuvre particulièrement stimulante par son interdisciplinarité, qui invite à dépasser le compartimentage universitaire actuel des sciences humaines et sociales et à résister à une certaine propension " positiviste » qui les conduit parfois à mimer, faute

Documents pour l'histoire du français langue étrangère ou seconde, 52 | 2014 
de sérieusement les imiter, les sciences de la nature. Et reste, dans la littérature actuelle de ces sciences encore trop souvent fascinées par le paradigme "sémiotique ", une œuvre rare, en ce que ses analyses ne sont dissociées ni de l'histoire des savoirs étudiés ni de l'historicité de son auteur.

\section{AUTEUR}

\section{HENRI BESSE}

ENS de Lyon 